\title{
Graphical Analysis of Kampé De Fériet's Series with Implementation of MATLAB
}

\author{
Praveen Agarwal \\ Department of Mathematics \\ Anand International College of Engineering \\ Jaipur-303012, India.
}

\author{
Mehar Chand \\ Department of Mathematics \\ Malwa College of IT and Management \\ Bathinda-151001, India.
}

\begin{abstract}
In the present Era, MATLAB has become a standard instructional tool for introductory and advanced courses in Mathematics, Engineering, and Science. In industry, MATLAB is now the tool of choice for high-productivity research, development and analysis. It is widely applied in applied algebra, statistics processing, automation and digital communication. In this paper, the Kampé de Fériet's series is analyzed and interpreted. The remarkable thing of this paper is the crucial MATLAB coding for Kampé de Fériet's series using the MATLAB(R2012a) and interpretation has also be taken on database as well as graphical for different parameters.
\end{abstract}

\section{General Terms}

Hypergeometric function, Special function.

\section{Keywords}

Kampé de Fériet's series, Special function, Matlab.

\section{INTRODUCTION}

Currently, more than thirty soft-wares are widely applied in mathematics. These mathematics soft-wares can be divided into two categories in terms of their function:

- The first category including Mathematica and Maple features on mathematical analysis, symbolic computation, formula reasoning and are capable of computing the analytical solution to mathematical problems while the disadvantage of these software are that they are not efficient when it comes to processing large quantity of data.

- The second category is the numerical computing soft-wares including Matlab, XPASS and GAUSS etc. The advantages of these soft-wares are that they are efficient to process large quantity of data.

Now a days, the core part of the first category softwares have been incorporated into the second category, say Matlab, and that makes Matlab become the main stream software in the field of mathematics. In the international academic community, Matlab has been recognized as accurate and reliable scientific computing standard software. In the design of research units and industry, Matlab is considered the first choice for research and development soft-ware tools.

In recent years, with the introduction of new media products, there has been a shift in the use of programming languages from FORTRAN or C to Matlab for implementing numerical methods. Over the years, many papers have been written on the subject of numerical methods. The main benefit is that one doesn't have to know the mathematical theory in order to apply the numerical methods for solving their real-life problems. Matlab stands for MATRIX LABORATARY (Matrix Laboratory), which was first used by Dr. Cleve Moler in New Mexico University United States to teach courses of linear algebra. The basic data unit is a matrix without dimension restriction.

In Matlab, the matrix computing becomes extremely easy. Dr. Moler in 1984 launched the official version of the soft-ware, in the later editions, he also gradually added to the control system, system identification, signal processing and communications, more than a toolbox, so Matlab became to be widely used in automatic control, image signal processing, biomedical engineering, speech processing, radar engineering, signal analysis, optimization and other fields. It has the following functions and features:

- Efficient numerical computation and symbolic computation capabilities, enables us to shift from the analysis of complex mathematical operations freed to complete graphics, and from programming to achieve the results visualization.

- Feature-rich application of the toolbox, providing a large number of convenient and practical processing tools.

- Friendly interface and close to the mathematical expression of the naturalization language, easy to learn and master.

In pure mathematics, since Matlab is an integrated computer software which has three functions: symbolic computing, numerical computing and graphics drawing. Matlab is capable to carry out many functions including computing polynomials and rational polynomials, solving equations and computing many kinds of mathematical expressions. One can also use Matlab to calculate the limit, derivative, integral and Taylor series of some mathematical expressions. With Matlab, The graphs of functions with one or two variables can be easily drawn in selected domain. Therefore, functions can be studied by visualization for their main Characteristics. Matlab is also a system which can be easily expanded. Matlab provides many powerful software packages which can be easily incorporated into the clients system.

The distinct scientific communities that are working on various aspects of automatic analysis of data include Combinatorial Pattern Matching, Data Mining, Computational Statistics, Network Analysis, Text Mining, Image Processing, Syntactical Pattern Recognition, Machine Learning, Statistical Pattern Recognition, Computer Vision, and many others. Dwork B. [4] and Yoshida Masaaki [14] worked on generalized Hypergeometric Functions . Watanabe Y et al [13] deals with a fast structural matching and its application to pattern analysis of 2-D electrophoresis images. Another important work of Rumshisky et al [11] deals with inducing sense-discriminating context patterns from sense-tagged corpora. One can also quote the work of Bevel Tom et al [2] on bloodstain pattern analysis with an introduction to crime scene reconstruction and of Hui Wang et al [10] for highdefinition metrology based spatial variation pattern analysis for the dynamic pattern analysis framework to the analysis of spatial-temporal crime relationships. The important work on 
MATLAB of Stormy Attaway[12] can be seen. Askey, R. A. [1] and Daalhuis, Adri B. Olde [3] also worked on generalized hypergeometric function.

The computation of the Kampé de Fériet's Series, a special function encountered in a variety of applications, is frequently sought. However, aside from the most basic hypergeometric functions, this is an extremely difficult task in practice. The reason for this is that the non-trivial structure of the series that defines the function creates many numerical issues such as cancellation and round-off error, which becomes especially significant for certain ranges of the parameters and the variables. This results in many methods of numerical computation being ineffective for all but the simplest parameter and variable ranges.

In this paper, main focus on computing Kampé de Fériet's Sereis of two variable $F_{l: m ; n}^{p: q ;}\left[\begin{array}{l}\left(a_{p}\right):\left(b_{q}\right) ;\left(c_{k}\right) ; x \\ \left(\alpha_{l}\right):\left(\beta_{m}\right) ;\left(\gamma_{n}\right) ;\end{array}\right]$ for different parameters. The program which will be used to compute these functions will be MATLAB (R2012a), which employs double precision arithmetic, so it is especially important for effective methods to be found to overcome the numerical issues involved.

Just as the Gaussian series ${ }_{2} F_{1}$ was generalized to ${ }_{p} F_{q}$ by increasing the number of the numerator and denominator parameters, the four Appell series were undefined and generalized by Kampé de Fériet Series who defined a general hypergeometric series in two variables. In 1921, Kampé de Fériet and studied the function named after him. Using the notation introduces in 1976 by Srivastava and Panda, Kampe de Feriet's series is defined as [8]:

$$
\begin{aligned}
& F_{l: m ; n}^{p: q ; k}\left[\begin{array}{l}
\left(a_{p}\right):\left(b_{q}\right) ;\left(c_{k}\right) ; x, y \\
\left(\alpha_{l}\right):\left(\beta_{m}\right) ;\left(\gamma_{n}\right) ;
\end{array}\right] \\
& =\sum_{r, s=0}^{\infty} \frac{\prod_{j=1}^{p}\left(a_{j}\right)_{r+s} \prod_{j=1}^{q}\left(b_{j}\right)_{r} \prod_{j=1}^{k}\left(c_{j}\right)_{s}}{\prod_{j=1}} \frac{x^{r}}{r} \prod_{j=1}^{m}\left(\beta_{j}\right)_{r} \prod_{j=1}^{n}\left(\gamma_{j}\right)_{s} \\
& r !
\end{aligned}
$$

which will converge under the following conditions:

$$
\begin{aligned}
& p+q<l+m+1, p+k<l+n+1,|x|<\infty,|y|<\infty \\
& p+q=l+m+1, p+k=l+n+1
\end{aligned}
$$

Srivastavs and Karlsson [6], Srivastava and Mnocha [7] and Exton [9] introduced many properties and functions of Kampé de Fériet function. Srivastava and Karlsson [6] list only 20 nontrivial instances in which Kampé de Fériet functions can be expressed in terms of generalized hypergeometric functions. Exton and Krupnikov [5] have derived a large collection of formulas involving this function.

In our investigation, we take the parameters for Kampé de Fériet's Sereis as following:

If $p=q=k=l=m=n=3$ the equation (1) reduces to:

$$
\begin{aligned}
& F_{3: 3 ; 3}^{3: 33 ;}\left[\begin{array}{l}
\left(a_{3}\right):\left(b_{3}\right) ;\left(c_{3}\right) ; x, y \\
\left(\alpha_{3}\right):\left(\beta_{3}\right) ;\left(\gamma_{3}\right) ;
\end{array}\right] \\
& =\sum_{r, s=0}^{\infty} \frac{\prod_{j=1}^{3}\left(a_{j}\right)_{r+s} \prod_{j=1}^{3}\left(b_{j}\right)_{r} \prod_{j=1}^{3}\left(c_{j}\right)_{s+s}}{\prod_{j=1}^{3}\left(\beta_{j}\right)_{r} \prod_{j=1}^{3}\left(\gamma_{j}\right)_{s}} \frac{x^{r}}{r !} \frac{y^{s}}{s !}
\end{aligned}
$$

If $p=2, q=3, k=2, l=3, m=2, n=3$ the equation reduces to:

$$
\begin{aligned}
& F_{3: 2 ; 3}^{2: 3 ; 2}\left[\begin{array}{l}
\left(a_{2}\right):\left(b_{3}\right) ;\left(c_{2}\right) ; \\
\left(\alpha_{3}\right):\left(\beta_{2}\right) ;\left(\gamma_{3}\right) ;
\end{array}, y\right] \\
& =\sum_{r, s=0}^{\infty} \frac{\prod_{j=1}^{2}\left(a_{j}\right)_{r+s} \prod_{j=1}^{3}\left(b_{j}\right)_{r} \prod_{j=1}^{2}\left(c_{j}\right)_{s}}{\prod_{j=1}^{3}\left(\alpha_{j}\right)_{r+s} \prod_{j=1}^{2}\left(\beta_{j}\right)_{r} \prod_{j=1}^{3}\left(\gamma_{j}\right)_{s}} \frac{x^{r}}{r !} \frac{y^{s}}{s !}
\end{aligned}
$$

In this paper, main work is interpretation and analysis of Kampé de Fériet's Sereis of two variables on the basis of database and graphical for particular parameters as assigned for equation (2) and (3). In section second, a program is made to establish the database for equation (2) and (3). In third section using the program, database for different parameters of $F_{3: 2 ; 3 ;}^{2: 3 ; 2}\left[\begin{array}{l}\left(a_{2}\right):\left(b_{3}\right) ;\left(c_{2}\right) ; \\ \left(\alpha_{3}\right):\left(\beta_{2}\right) ;\left(\gamma_{3}\right) ;\end{array}, y, y\right]$ and $F_{3: 3 ; 3}^{33 ; 3}\left[\begin{array}{l}\left(a_{3}\right):\left(b_{3}\right) ;\left(c_{3}\right) ; \\ \left(\alpha_{3}\right):\left(\beta_{3}\right) ;\left(\gamma_{3}\right) ;\end{array}, y\right]$ are established. In the fourth section, graphs are made corresponding to the database and in fifth section, mesh-plot of Kampé de Fériet's Sereis for different parameters. In sixth section, interpretation and comparison are made on the basis of databases as well as graphs established in section third, fourth and fifth. Also, the time taken by the each graph in MATLAB is calculated.

\section{PROGRAMMING OF THE KAMPE DE FERIET SERIES IN MATLAB}

Code is divided in parts as Kampe de Feriet Series involves Pochhammer's symbol $(\mathrm{e})_{\mathrm{r}}=\Gamma(\mathrm{e}+\mathrm{r}) / \Gamma(\mathrm{e})$.

(a) Program Kampe de Feriet series for equation (2):

Code of Kampe de Feriet series for equation (2):

Part (1)

function $[\mathrm{e}]=\mathrm{eu}(\mathrm{a} 1, \mathrm{r} 1, \mathrm{a} 2, \mathrm{r} 2, \mathrm{a} 3, \mathrm{r} 3)$

$\%$ Pochhammer's symbol (e)r=gamma(e+r)/gamma(e)

$\%$ we cosider here

eu(a1,r1, a2,r2,a3,r3) $=($ gamma $(\mathrm{a} 1+\mathrm{r} 1) \cdot /$ gamma(a1) $)(\operatorname{gamma}(\mathrm{a} 2$ $+\mathrm{r} 2)$

./gamma(a2))(gamma(a3+r3)./gamma(a3))

$\mathrm{e} 1=\operatorname{gamma}(\mathrm{a} 1+\mathrm{r} 1) \cdot / \operatorname{gamma}(\mathrm{a} 1)$;

$\mathrm{e} 2$ = gamma $(\mathrm{a} 2+\mathrm{r} 2) \cdot / \operatorname{gamma}(\mathrm{a} 2)$;

e3 = gamma(a3+r3)./gamma(a3);

$\mathrm{e}=\mathrm{e} 1 . * \mathrm{e} 2 .{ }_{\mathrm{e}} 3$;

end

Code of Kampe de Feriet series for equation (2):

Part (2)

function $[\mathrm{Kn}]=$ Kampe $(\mathrm{a} 1, \mathrm{a} 2, \mathrm{a} 3, \mathrm{~b} 1, \mathrm{~b} 2, \mathrm{~b} 3, \mathrm{c} 1, \mathrm{c} 2, \mathrm{c} 3$,

d1,d2,d3, e1,e2,e3, f1,f2,f3, x,y) 
\%Graph of Kn(a1,a2,a3: b1,b2,b3, c1,c2,c3; d1,d2,d3: e1,e2,e3, f1,f2,f3, x,y)

$\%$ Kampe de Feriet series;

$\% \mathrm{~F}^{\wedge}(3: 3 ; 3) \_(3: 3 ; 3)[(\mathrm{a} 3):(\mathrm{b} 3) ;(\mathrm{c} 3) ;($ alpha3):(beta3);(gamma3); $\mathrm{x}, \mathrm{y}]=\operatorname{sum}(\mathrm{r}, \mathrm{s}=0: \operatorname{in} \% \mathrm{f}(\operatorname{prod}(\mathrm{j}=1: 3(\mathrm{aj})) \operatorname{prod}(\mathrm{j}=1: 3(\mathrm{bj})) \operatorname{prod}(\mathrm{j}=1$ : $3(\mathrm{cj})) /$

$\% \operatorname{prod}(\mathrm{j}=1: 3$ (alphaj) $) \operatorname{prod}(\mathrm{j}=1: 3($ alphaj $)) \operatorname{prod}(\mathrm{j}=1: 3$ (alphaj)) $\%\left(\mathrm{x}^{\wedge} \mathrm{r} \cdot \mathrm{y}^{\wedge} \mathrm{s} /\right.$ fatorial $\left.\left.\left.(\mathrm{r})(\mathrm{s})\right)\right)\right)$

$\mathrm{k}=0$;

for $\mathrm{r}=0: 15$

for $\mathrm{s}=0: 15$

$\mathrm{k}=\mathrm{k}+(\mathrm{eu}(\mathrm{a} 1, \mathrm{r}+\mathrm{s}, \mathrm{a} 2, \mathrm{r}+\mathrm{s}, \mathrm{a} 3, \mathrm{r}+\mathrm{s}) . * \mathrm{eu}(\mathrm{b} 1, \mathrm{r}, \mathrm{b} 2, \mathrm{r}, \mathrm{b} 3, \mathrm{r}) . * \mathrm{eu}(\mathrm{c}$ $1, \mathrm{~s}, \mathrm{c} 2, \mathrm{~s}, \mathrm{c} 3, \mathrm{~s})) \cdot *(\mathrm{x} . \wedge \mathrm{r}) \cdot *(\mathrm{y} \cdot \wedge \mathrm{s}) . /(($ factorial(r).*factorial(s)) . eu $(\mathrm{d} 1, \mathrm{r}+\mathrm{s}, \mathrm{d} 2, \mathrm{r}+\mathrm{s}, \mathrm{d} 3, \mathrm{r}+\mathrm{s}) . * \mathrm{eu}(\mathrm{e} 1, \mathrm{r}, \mathrm{e} 2, \mathrm{r}, \mathrm{e} 3, \mathrm{r}) . * \mathrm{eu}(\mathrm{f} 1, \mathrm{~s}, \mathrm{f}$ $2, \mathrm{~s}, \mathrm{f} 3, \mathrm{~s}))$;

end

end

$\mathrm{Kn}=\mathrm{k}$;

end

Plot Graph:

tic,plot3(x,y,k1,x,y,k2,x,y,k3,x,y,k4),toc;

legend('k1','k2','k3','k4')

(b)Program Kampe de Feriet series for equation (3):

Code of Kampe de Feriet series for equation (3):

Part (1)

function $[\mathrm{e}]=\mathrm{eu}(\mathrm{a} 1, \mathrm{r} 1, \mathrm{a} 2, \mathrm{r} 2, \mathrm{a} 3, \mathrm{r} 3)$

\%Pochhammer's symbol (e)r=gamma(e+r)/gamma(e)

$\%$ we cosider here

eu(a1,r1,a2,r2,a3,r3) $=($ gamma $(\mathrm{a} 1+\mathrm{r} 1) \cdot /$ gamma(a1) $)($ gamma $(\mathrm{a} 2$ $+\mathrm{r} 2)$

./gamma(a2))(gamma(a3+r3)./gamma(a3))

e1 = gamma(a1+r1)./gamma(a1);

e2 = gamma(a2+r2)./gamma(a2);

$\mathrm{e} 3=\operatorname{gamma}(\mathrm{a} 3+\mathrm{r} 3) \cdot / \operatorname{gamma}(\mathrm{a} 3)$;

$\mathrm{e}=\mathrm{e} 1 . * \mathrm{e} 2 . * \mathrm{e} 3$;

end

Code of Kampe de Feriet series for equation (3):

Part (2)

function $[\mathrm{e}]=\mathrm{eu} 1(\mathrm{a} 1, \mathrm{r} 1, \mathrm{a} 2, \mathrm{r} 2)$

\%Pochhammer's symbol (e)r=gamma(e+r)/gamma(e)

$\%$ we cosider here

$\mathrm{eu}(\mathrm{a} 1, \mathrm{r} 1, \mathrm{a} 2, \mathrm{r} 2)=(\operatorname{gamma}(\mathrm{a} 1+\mathrm{r} 1) \cdot / \operatorname{gamma}(\mathrm{a} 1))(\operatorname{gamma}(\mathrm{a} 2+\mathrm{r} 2) . /$ $\operatorname{gamma}(\mathrm{a} 2)))$

e1 = gamma(a1+r1)./gamma(a1);

e2 = gamma(a2+r2)./gamma(a2);

$\mathrm{e}=\mathrm{e} 1{ }^{*} \mathrm{e} 2$;

end

Code of Kampe de Feriet series for equation (3):

Part (3)

function $[\mathrm{Kn}]=\operatorname{Kampe} 1(\mathrm{a} 1, \mathrm{a} 2, \mathrm{~b} 1, \mathrm{~b} 2, \mathrm{~b} 3, \mathrm{c} 1, \mathrm{c} 2, \mathrm{~d} 1, \mathrm{~d} 2, \mathrm{~d} 3$, $\mathrm{e} 1, \mathrm{e} 2, \mathrm{f} 1, \mathrm{f} 2, \mathrm{f} 3, \mathrm{x}, \mathrm{y})$

\%Graph of Kn(a1,a2,a3: b1,b2,b3, c1,c2,c3; d1,d2,d3:

$\mathrm{e} 1, \mathrm{e} 2, \mathrm{e} 3, \mathrm{f} 1, \mathrm{f} 2, \mathrm{f} 3, \mathrm{x}, \mathrm{y})$

$\%$ Kampe de Feriet series;

$\% \mathrm{~F}^{\wedge}(2: 3 ; 2) \_(3: 2 ; 3)[(\mathrm{a} 2):(\mathrm{b} 3) ;(\mathrm{c} 2) ;($ alpha3):(beta2);(gamma3); $\mathrm{x}, \mathrm{y}]=\operatorname{sum}(\mathrm{r}, \mathrm{s}=0: \mathrm{in} \% \mathrm{f}(\operatorname{prod}(\mathrm{j}=1: 3(\mathrm{aj})) \operatorname{prod}(\mathrm{j}=1: 2(\mathrm{bj})) \operatorname{prod}(\mathrm{j}=1$ :

$3(\mathrm{cj}))$

$\% / \operatorname{prod}(\mathrm{j}=1: 3$ (alphaj))prod $(\mathrm{j}=1: 2($ alphaj $))$

$\% \operatorname{prod}(\mathrm{j}=1: 3($ alphaj $))\left(\mathrm{x}^{\wedge} \mathrm{r} \cdot \mathrm{y}^{\wedge} \mathrm{s} /\right.$ fatorial $\left.\left.\left.(\mathrm{r})(\mathrm{s})\right)\right)\right)$

$\mathrm{k}=0$;

for $r=0: 15$

for $\mathrm{s}=0: 15$ $\mathrm{k}=\mathrm{k}+\left(\mathrm{eu} 1(\mathrm{a} 1, \mathrm{r}+\mathrm{s}, \mathrm{a} 2, \mathrm{r}+\mathrm{s}) . * \mathrm{eu}(\mathrm{b} 1, \mathrm{r}, \mathrm{b} 2, \mathrm{r}, \mathrm{b} 3, \mathrm{r}) \cdot{ }^{*} \mathrm{eu} 1(\mathrm{c} 1\right.$ $, \mathrm{s}, \mathrm{c} 2, \mathrm{~s})) . *\left(\mathrm{x} .{ }^{\wedge} \mathrm{r}\right) \cdot{ }^{*}\left(\mathrm{y} .{ }^{\wedge} \mathrm{s}\right) . /\left((\right.$ factorial $(\mathrm{r}) . *$ factorial $(\mathrm{s})){ }^{*}$ $\mathrm{eu}(\mathrm{d} 1, \mathrm{r}+\mathrm{s}, \mathrm{d} 2, \mathrm{r}+\mathrm{s}, \mathrm{d} 3, \mathrm{r}+\mathrm{s}) . * \mathrm{*} u 1(\mathrm{e} 1, \mathrm{r}, \mathrm{e} 2, \mathrm{r}) . * \mathrm{*} u(\mathrm{f} 1, \mathrm{~s}, \mathrm{f} 2$, $\mathrm{s}, \mathrm{f} 3, \mathrm{~s})$ );

end

end

$\mathrm{Kn}=\mathrm{k}$;

end

Plot Graph:

tic,plot3(x,y,k1,x,y,k2,x,y,k3,x,y,k4),toc;

legend('k1','k2','k3','k4')

\section{DATABASE FOR KAMPE DE FERIET SERIES}

In this section, data-base for Kampé de Fériet's Series for the different parameters for the equation (2) as well as for equation (3) are established.

To establish Database of $F_{3: 3 ; 3}^{3: 3 ; 3}\left[\begin{array}{l}\left(a_{3}\right):\left(b_{3}\right) ;\left(c_{3}\right) ; \\ \left(\alpha_{3}\right):\left(\beta_{3}\right) ;\left(\gamma_{3}\right) ;\end{array} x, y\right]$ for

different parameters, it can be expressed as:

$k=k\left[a_{1}, a_{2}, a_{3}: b_{1}, b_{2}, b_{3} ; c_{1}, c_{2}, c_{3} ; \alpha_{1}, \alpha_{2}, \alpha_{3}: \beta_{1}, \beta_{2}, \beta_{3} ; \gamma_{1}, \gamma_{2}, \gamma_{3} ; x, y\right]$

Command window for Database (1):

$>\mathrm{x}=(-5: 1: 5)^{\prime}$

$>\mathrm{y}=(-5: 1: 5)^{\prime}$

$>>\mathrm{k} 1=\operatorname{Kampe}(1,1,1,1,1,1,1,1,1,1,1,1,1,1,1,1,1,1, \mathrm{x}, \mathrm{y})$;

$>>\mathrm{k} 2=$ Kampe $(3,3,3,3,3,3,3,3,3,3,3,3,3,3,3,3,3,3, \mathrm{x}, \mathrm{y})$;

$>>\mathrm{k} 3=\operatorname{Kampe}(5,5,5,5,5,5,5,5,5,5,5,5,5,5,5,5,5,5, \mathrm{x}, \mathrm{y})$

$>\mathrm{k} 4=$ Kampe $(7,7,7,7,7,7,7,7,7,7,7,7,7,7,7,7,7,7, \mathrm{x}, \mathrm{y})$

>> [x y k1 k2 k3 k4]

Table (1)

\begin{tabular}{|cccccc|}
\hline $1.0 \mathrm{e}+04 *$ & & & & & \\
$\mathrm{x}$ & $\mathrm{y}$ & $\mathrm{k} 1$ & $\mathrm{k} 2$ & $\mathrm{k} 3$ & $\mathrm{k} 4$ \\
-0.0005 & -0.0005 & 0.0000 & 0.0000 & 0.0000 & 0.0000 \\
-0.0004 & -0.0004 & 0.0000 & 0.0000 & 0.0000 & 0.0000 \\
-0.0003 & -0.0003 & 0.0000 & 0.0000 & 0.0000 & 0.0000 \\
-0.0002 & -0.0002 & 0.0000 & 0.0000 & 0.0000 & 0.0000 \\
-0.0001 & -0.0001 & 0.0000 & 0.0000 & 0.0000 & 0.0000 \\
0 & 0 & 0.0001 & 0.0001 & 0.0001 & 0.0001 \\
0.0001 & 0.0001 & 0.0007 & 0.0007 & 0.0007 & 0.0007 \\
0.0002 & 0.0002 & 0.0055 & 0.0055 & 0.0055 & 0.0055 \\
0.0003 & 0.0003 & 0.0403 & 0.0403 & 0.0403 & 0.0403 \\
0.0004 & 0.0004 & 0.2981 & 0.2981 & 0.2981 & 0.2981 \\
0.0005 & 0.0005 & 2.2023 & 2.2023 & 2.2023 & 2.2023
\end{tabular}

Command window for Database (2):

$>\mathrm{x}=(-5: 1: 5)^{\prime}$;

$>\mathrm{y}=(-5: 1: 5)^{\prime}$

$>>\mathrm{k} 1=\operatorname{Kampe}(1,1,1,2,2,2,3,3,3,4,4,4,5,5,5,6,6,6, \mathrm{x}, \mathrm{y})$;

$>>\mathrm{k} 2=\operatorname{Kampe}(3,3,3,4,4,4,5,5,5,6,6,6,7,7,7,8,8,8, \mathrm{x}, \mathrm{y})$

$>$ k3=Kampe $(5,5,5,7,7,7,8,8,8,9,9,9,10,10,10,11,11,11, \mathrm{x}, \mathrm{y})$;

$>$ > $\mathrm{x}$ y k1 k2 k3] 
Table (2)

\begin{tabular}{|ccccc|}
\hline $\mathrm{x}$ & $\mathrm{y}$ & $\mathrm{k} 1$ & $\mathrm{k} 2$ & $\mathrm{k} 3$ \\
-5.0000 & -5.0000 & 0.9858 & 0.7808 & 0.5667 \\
-4.0000 & -4.0000 & 0.9885 & 0.8178 & 0.6298 \\
-3.0000 & -3.0000 & 0.9913 & 0.8579 & 0.7025 \\
-2.0000 & -2.0000 & 0.9942 & 0.9013 & 0.7867 \\
-1.0000 & -1.0000 & 0.9971 & 0.9485 & 0.8849 \\
0 & 0 & 1.0000 & 1.0000 & 1.0000 \\
1.0000 & 1.0000 & 1.0030 & 1.0564 & 1.1359 \\
2.0000 & 2.0000 & 1.0060 & 1.1184 & 1.2974 \\
3.0000 & 3.0000 & 1.0091 & 1.1868 & 1.4910 \\
4.0000 & 4.0000 & 1.0122 & 1.2626 & 1.7249 \\
50000 & 50000 & 10154 & 13471 & 2.0107 \\
\hline
\end{tabular}

Command window for Database (3):

$>\mathrm{x}=(-5: 1: 5)^{\prime}$

$>\mathrm{y}=(-5: 1: 5)^{\prime}$

$>>\mathrm{k} 1=\operatorname{Kampe}(1,2,3,4,5,6,4,5,6,7,8,9,7,8,9,10,11,12, \mathrm{x}, \mathrm{y})$; $>>\mathrm{k} 2=$

Kampe(4,5,6,7,8,9,7,8,9,10,11,12,10,11,12,13,14,15,x,y); $>\mathrm{k} 3=$

Kampe $(7,8,9,7,8,9,10,11,12,10,11,12,13,14,15,16,17,18, \mathrm{x}, \mathrm{y})$; $>\mathrm{k} 4=$

Kampe $(7,8,9,10,11,12,10,11,12,13,14,15,16,17,18,19,20,21, x$, y);

$>$ > $\mathrm{x}$ y k1 k2 k3 k4]

Table (3)

\begin{tabular}{|cccccc|}
\hline $\mathrm{x}$ & $\mathrm{y}$ & $\mathrm{k} 1$ & $\mathrm{k} 2$ & $\mathrm{k} 3$ & $\mathrm{k} 4$ \\
-5.0000 & -5.0000 & 0.9810 & 0.7832 & 0.4447 & 0.6807 \\
-4.0000 & -4.0000 & 0.9847 & 0.8208 & 0.5185 & 0.7332 \\
-3.0000 & -3.0000 & 0.9885 & 0.8611 & 0.6070 & 0.7908 \\
-2.0000 & -2.0000 & 0.9923 & 0.9041 & 0.7137 & 0.8540 \\
-1.0000 & -1.0000 & 0.9961 & 0.9503 & 0.8428 & 0.9235 \\
0 & 0 & 1.0000 & 1.0000 & 1.0000 & 1.0000 \\
1.0000 & 1.0000 & 1.0039 & 1.0535 & 1.1924 & 1.0844 \\
2.0000 & 2.0000 & 1.0079 & 1.1111 & 1.4292 & 1.1777 \\
3.0000 & 3.0000 & 1.0120 & 1.1734 & 1.7227 & 1.2811 \\
4.0000 & 4.0000 & 1.0161 & 1.2408 & 2.0886 & 1.3957 \\
5.0000 & 5.0000 & 1.0202 & 1.3140 & 2.5481 & 1.5233 \\
\hline
\end{tabular}

(b) Database of $F_{32 ; 3 ;}^{23: 2}\left[\begin{array}{l}\left(a_{2}\right):\left(b_{3}\right) ;\left(c_{2}\right) ; \\ \left(\alpha_{3}\right):\left(\beta_{2}\right) ;\left(\gamma_{3}\right) ;\end{array} x, y\right.$ for different parameters:

$k=k\left[a_{1}, a_{2}: b_{1}, b_{2}, b_{3} ; c_{1}, c_{2} ; \alpha_{1}, \alpha_{2}, \alpha_{3}: \beta_{1}, \beta_{2} ; \gamma_{1}, \gamma_{2}, \gamma_{3} ; x, y\right]$

Command window for Database (4):

$>\mathrm{x}=(-1: .2: 1)^{\prime}$;

$>\mathrm{y}=(-1: .2: 1)^{\prime}$

$>>\mathrm{k} 1=\operatorname{Kampe} 1(1,1,1,1,1,1,1,1,1,1,1,1,1,1,1, \mathrm{x}, \mathrm{y})$; $>$ k2=Kampe1(3,3, 3,3,3, 3,3, 3,3,3, 3,3, 3,3,3, x,y); $>>\mathrm{k} 3=\operatorname{Kampe} 1(5,5,5,5,5,5,5,5,5,5,5,5,5,5,5, \mathrm{x}, \mathrm{y})$; >>k4=Kampe1(7,7, 7,7,7, 7,7, 7,7,7, 7,7, 7,7,7, x,y); > [x y k1 k2 k3 k4]
Table (4)

\begin{tabular}{|cccccr|}
\hline $\mathrm{x}$ & $\mathrm{y}$ & $\mathrm{k} 1$ & $\mathrm{k} 2$ & $\mathrm{k} 3$ & $\mathrm{k} 4$ \\
-1.0000 & -1.0000 & -0.1759 & 0.3163 & 0.3506 & 0.3594 \\
-0.8000 & -0.8000 & -0.0409 & 0.4013 & 0.4330 & 0.4412 \\
-0.6000 & -0.6000 & 0.1340 & 0.5069 & 0.5343 & 0.5416 \\
-0.4000 & -0.4000 & 0.3580 & 0.6377 & 0.6589 & 0.6646 \\
-0.2000 & -0.2000 & 0.6421 & 0.7997 & 0.8120 & 0.8153 \\
0 & 0 & 1.0000 & 1.0000 & 1.0000 & 1.0000 \\
0.2000 & 0.2000 & 1.4482 & 1.2474 & 1.2309 & 1.2263 \\
0.4000 & 0.4000 & 2.0069 & 1.5527 & 1.5143 & 1.5035 \\
0.6000 & 0.6000 & 2.7009 & 1.9292 & 1.8621 & 1.8430 \\
0.8000 & 0.8000 & 3.5604 & 2.3930 & 2.2889 & 2.2587 \\
1.0000 & 1.0000 & 4.6223 & 2.9642 & 2.8123 & 2.7679 \\
\hline
\end{tabular}

Command window for Database (5):

$>\mathrm{x}=(-10: 2: 10)^{\prime}$;

$>\mathrm{y}=(-10: 2: 10)^{\prime}$;

$>>\mathrm{k} 1=$ Kampe $1(1,2,3,4,5,6,4,5,6,7,8,9,7,8,9, \mathrm{x}, \mathrm{y})$;

$>\mathrm{k} 2=$ Kampe1 $(4,5,6,7,8,9,7,8,9,10,11,12,10,11,12, \mathrm{x}, \mathrm{y})$; $>>\mathrm{k} 3=$ Kampe1 $(7,8,9,7,8,9,10,11,12,10,11,12,13,14,15, \mathrm{x}, \mathrm{y})$; $>>\mathrm{k} 4=$

Kampe1(7,8,9,10,11,12,10,11,12,13,14,15,16,17,18,x,y); $>>[x$ y k1 k2 k3 k4]

Table (5)

\begin{tabular}{|cccccc|}
\hline $\mathrm{x}$ & $\mathrm{y}$ & $\mathrm{k} 1$ & $\mathrm{k} 2$ & $\mathrm{k} 3$ & $\mathrm{k} 4$ \\
-10.0000 & -10.0000 & 0.9249 & 0.5281 & 0.2421 & 0.2553 \\
-8.0000 & -8.0000 & 0.9387 & 0.5932 & 0.3120 & 0.3270 \\
-6.0000 & -6.0000 & 0.9530 & 0.6698 & 0.4076 & 0.4237 \\
-4.0000 & -4.0000 & 0.9680 & 0.7606 & 0.5404 & 0.5561 \\
-2.0000 & -2.0000 & 0.9836 & 0.8691 & 0.7284 & 0.7400 \\
0 & 0 & 1.0000 & 1.0000 & 1.0000 & 1.0000 \\
2.0000 & 2.0000 & 1.0172 & 1.1596 & 1.4013 & 1.3744 \\
4.0000 & 4.0000 & 1.0353 & 1.3565 & 2.0093 & 1.9247 \\
6.0000 & 6.0000 & 1.0544 & 1.6026 & 2.9563 & 2.7518 \\
8.0000 & 8.0000 & 1.0745 & 1.9148 & 4.4769 & 4.0254 \\
10.0000 & 10.0000 & 1.0959 & 2.3176 & 7.0022 & 6.0401 \\
\hline
\end{tabular}

\section{GRAPHS BASED ON THE DATA- BASE}

In this section, for different parameters of Kampé de Fériet's Series taken as to establish the data-base in table (1), (2), (3), (4) and (5) in section (3) corresponding graphs are made in figure (1), (2), (3), (4) and (5). Elapsed time for graphs (1), (2), (3), (4) and (5) are 0.110104 seconds, 0.309434 seconds, 0.145947 seconds, 0.101553 seconds and 0.103613 seconds respectively. 


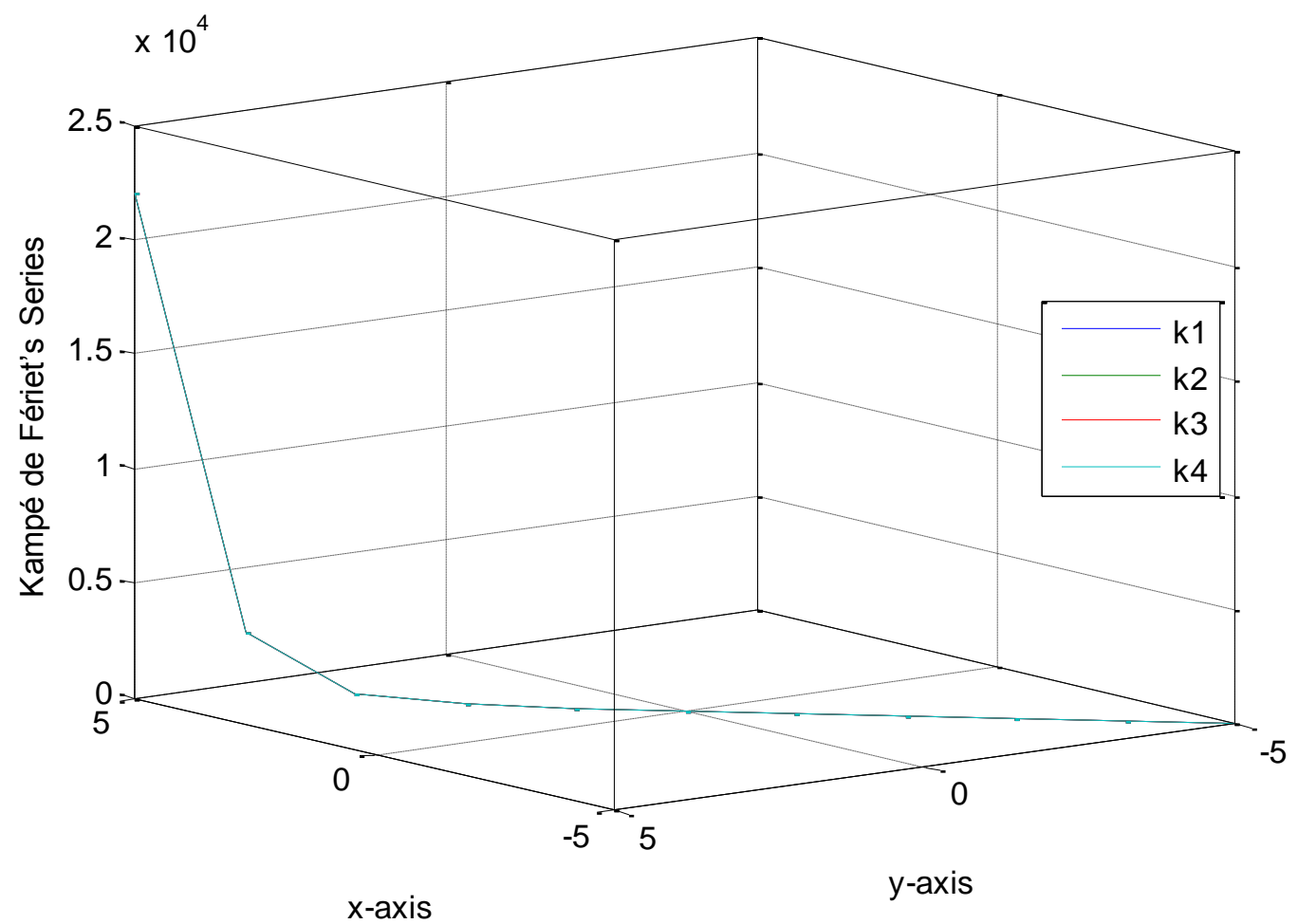

Fig.1 Based on table (1)

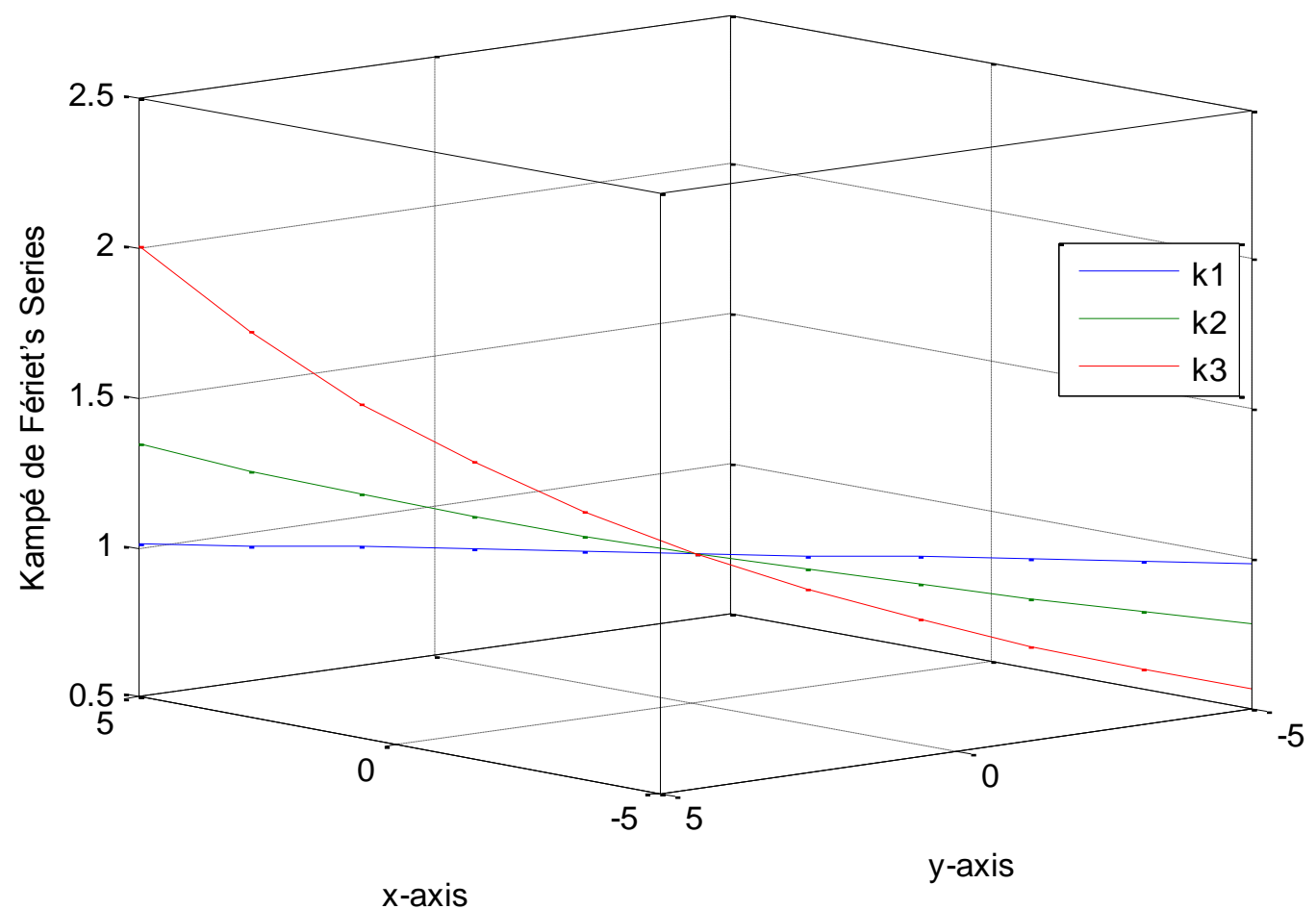

Fig.2 Based on table (2) 


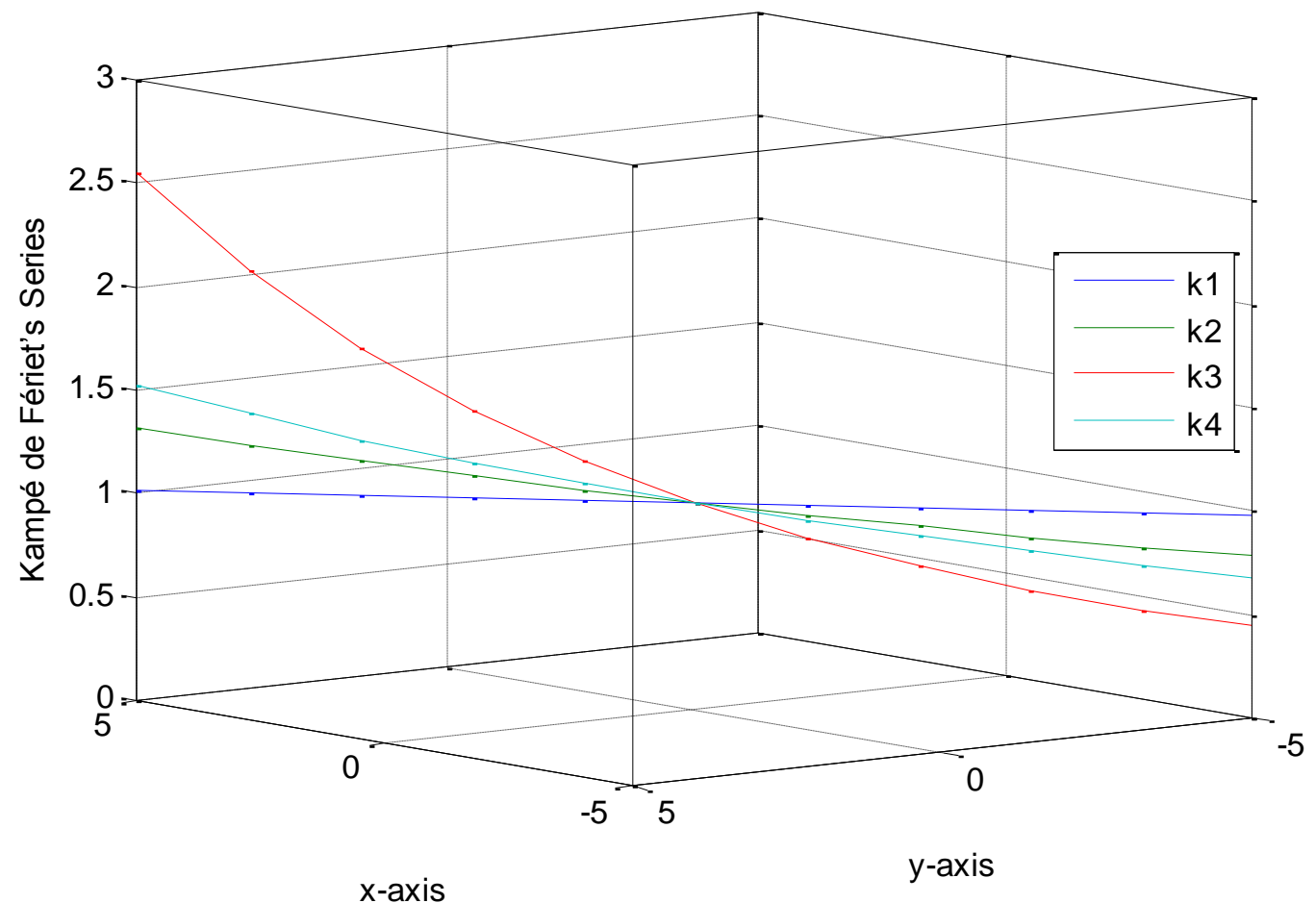

Fig.3 Based on table (3)

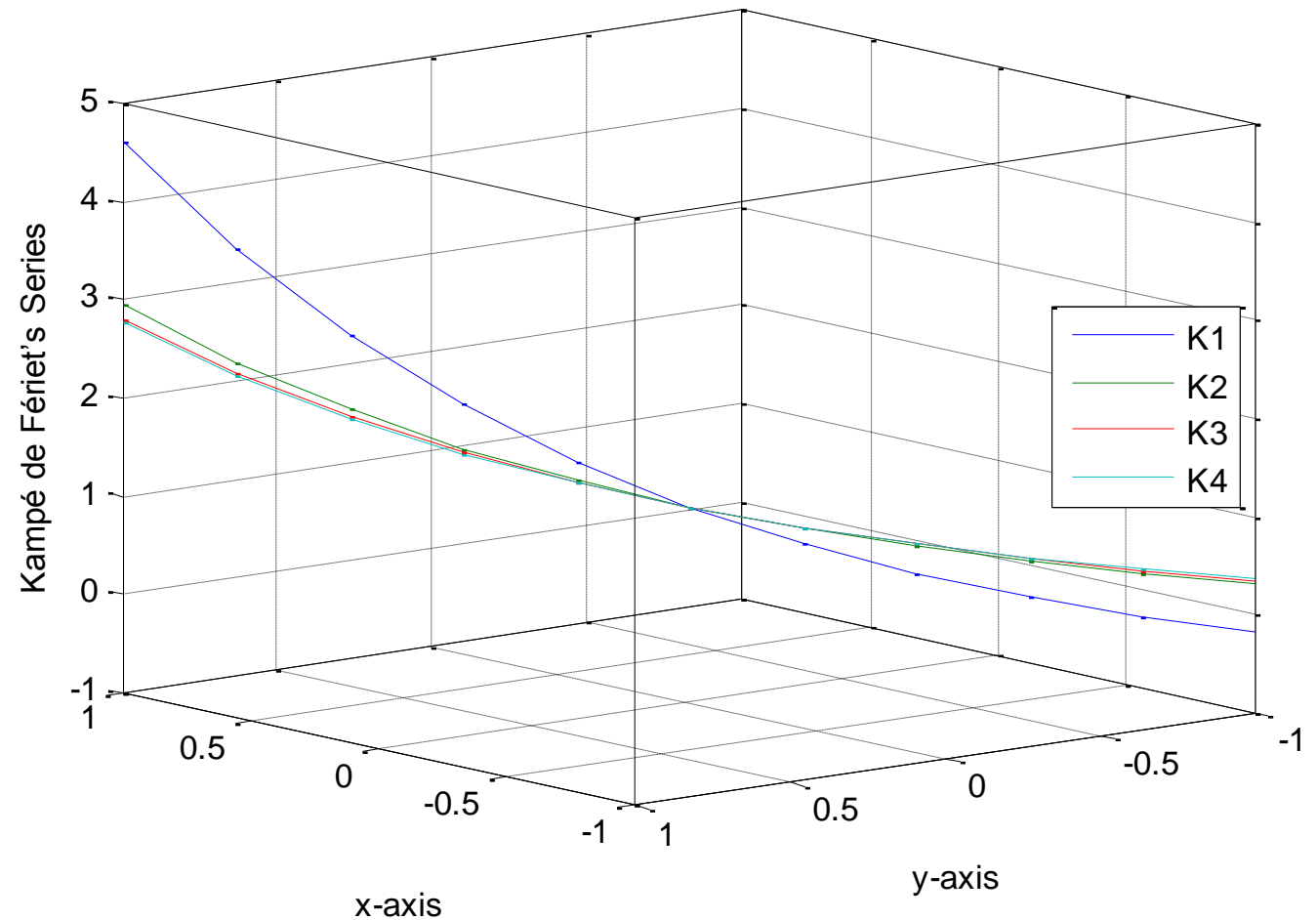

Fig.4 Based on table (4) 


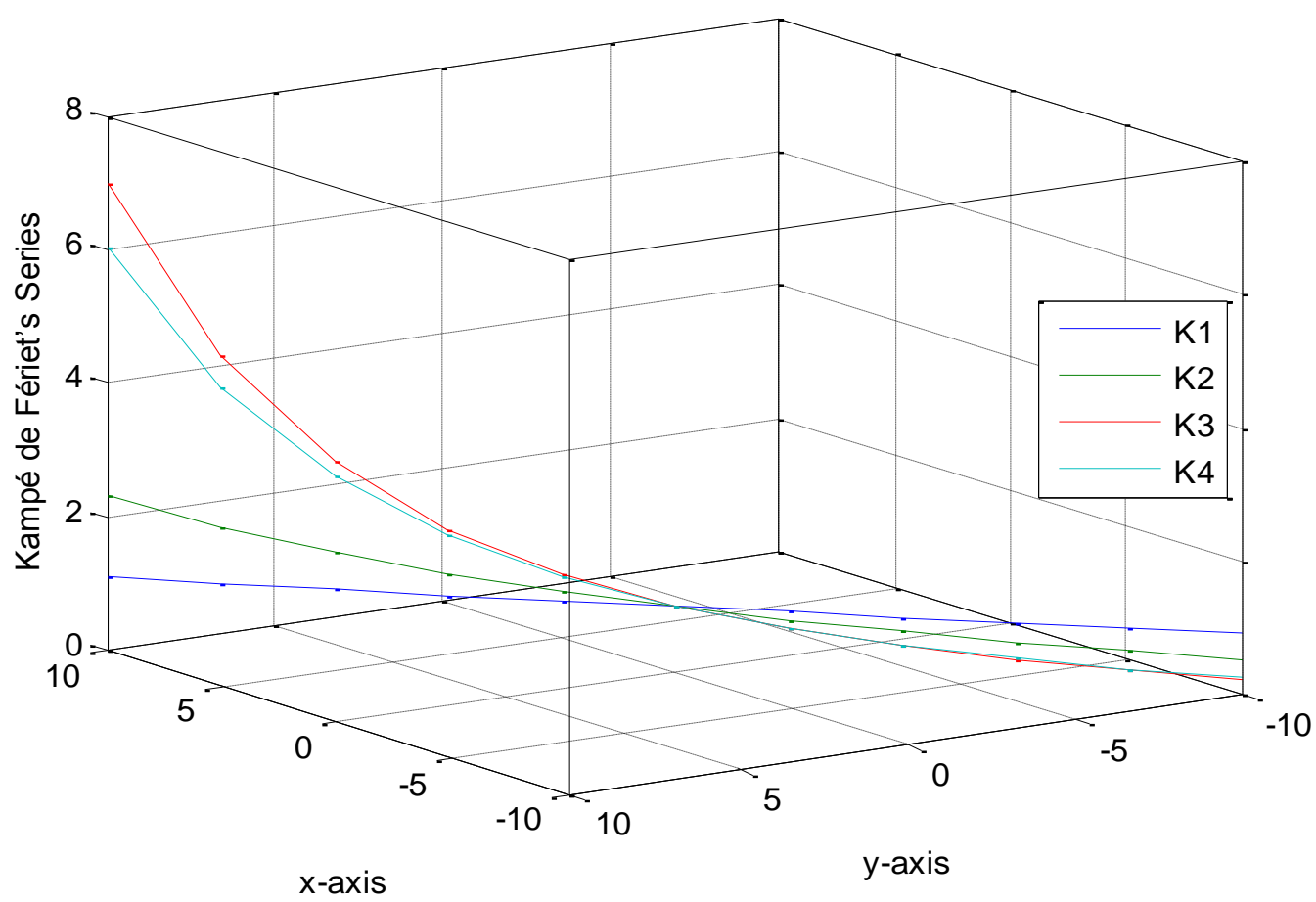

Fig.5 Based on table (5)

\section{MESH-PLOTS OF KAMPE DE} FERIET'S SERIES:

(a) Command window:

$>$ > $\mathrm{x}$ y] $=$ meshgrid(-5:1:5,-5:1:5);

> k1= Kampe $(1,1,1,2,2,2,3,3,3,4,4,4,5,5,5,6,6,6, \mathrm{x}, \mathrm{y})$;

> tic, mesh(x,y,k1),toc

Elapsed time is 0.101553 seconds. (b) Command window:

> $[\mathrm{x} \mathrm{y}]=\operatorname{meshgrid}(-5: 1: 5,-5: 1: 5)$;

$>\mathrm{k} 1=$

Kampe(1,2,3,4,5,6,4,5,6,7,8,9,7,8,9,10,11,12,x,y);

> tic, mesh(x,y,k1),toc

Elapsed time is 0.103613 seconds.

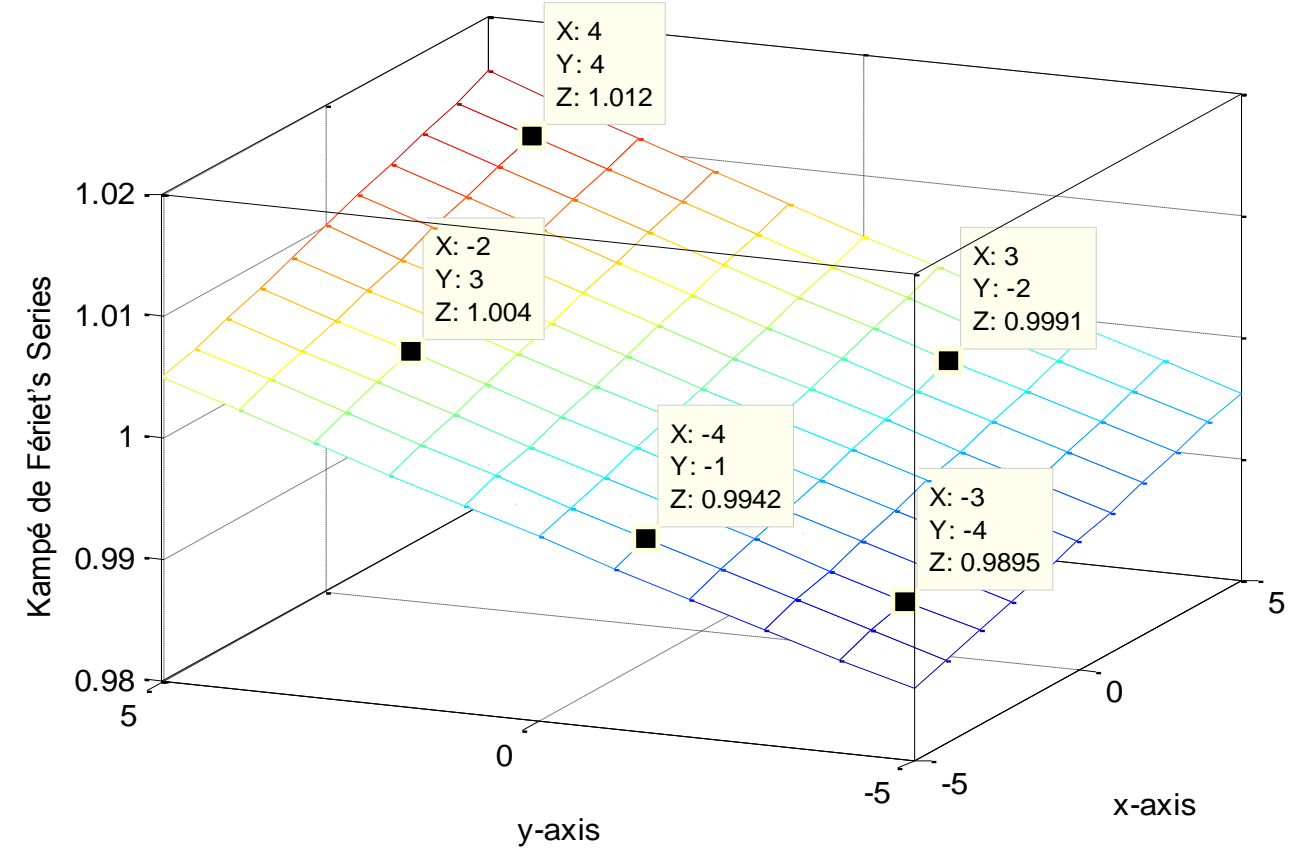

Fig.6 


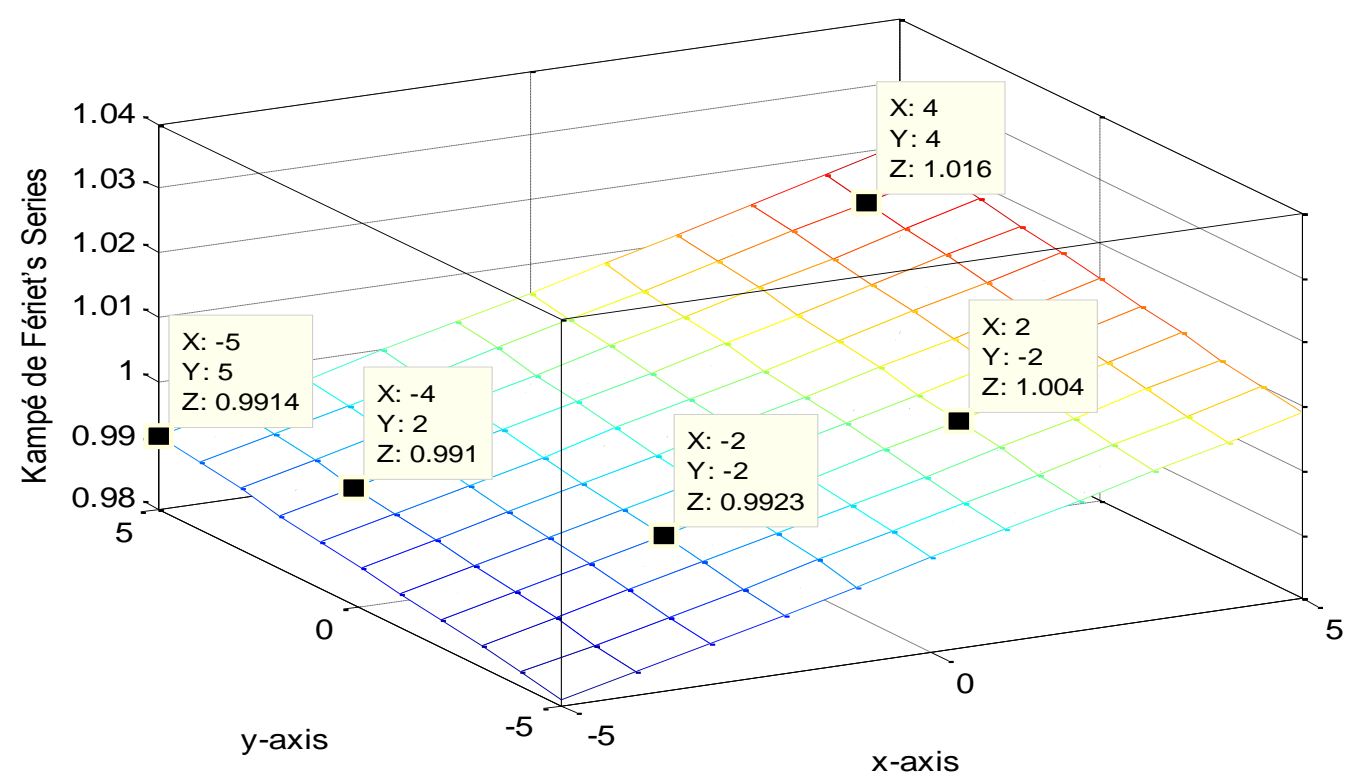

Fig.7

\section{GRAPH FOR CIRCULAR POINTS OF KAMPE DE FERIET'S SERIES:}

Command window:

$>$ t=-pi:pi/100:pi;

$>\mathrm{x}=\cos (\mathrm{t})$;

$>\mathrm{y}=\sin (\mathrm{t})$

>>k1=Kampe $(1,2,3,4,5,6,4,5,6,7,8,9,7,8,9,10,11,12, \mathrm{x}, \mathrm{y})$;
>>k2=Kampe $(4,5,6,7,8,9,7,8,9,10,11,12,10,11,12,13,14,15, \mathrm{x}$, y);

$>>\mathrm{k} 3=\operatorname{Kampe}(7,8,9,7,8,9,10,11,12,10,11,12,13,14,15,16,17,1$ $8, \mathrm{x}, \mathrm{y})$

>>k4=Kampe $(7,8,9,10,11,12,10,11,12,13,14,15,16,17,18,19,2$

$0,21, \mathrm{x}, \mathrm{y})$;

> tic,plot3(x,y,k1,x,y,k2,x,y,k3,x,y,k4),toc;

> legend('k1','k2','k3','k4')

Elapsed time is 0.230064 seconds.

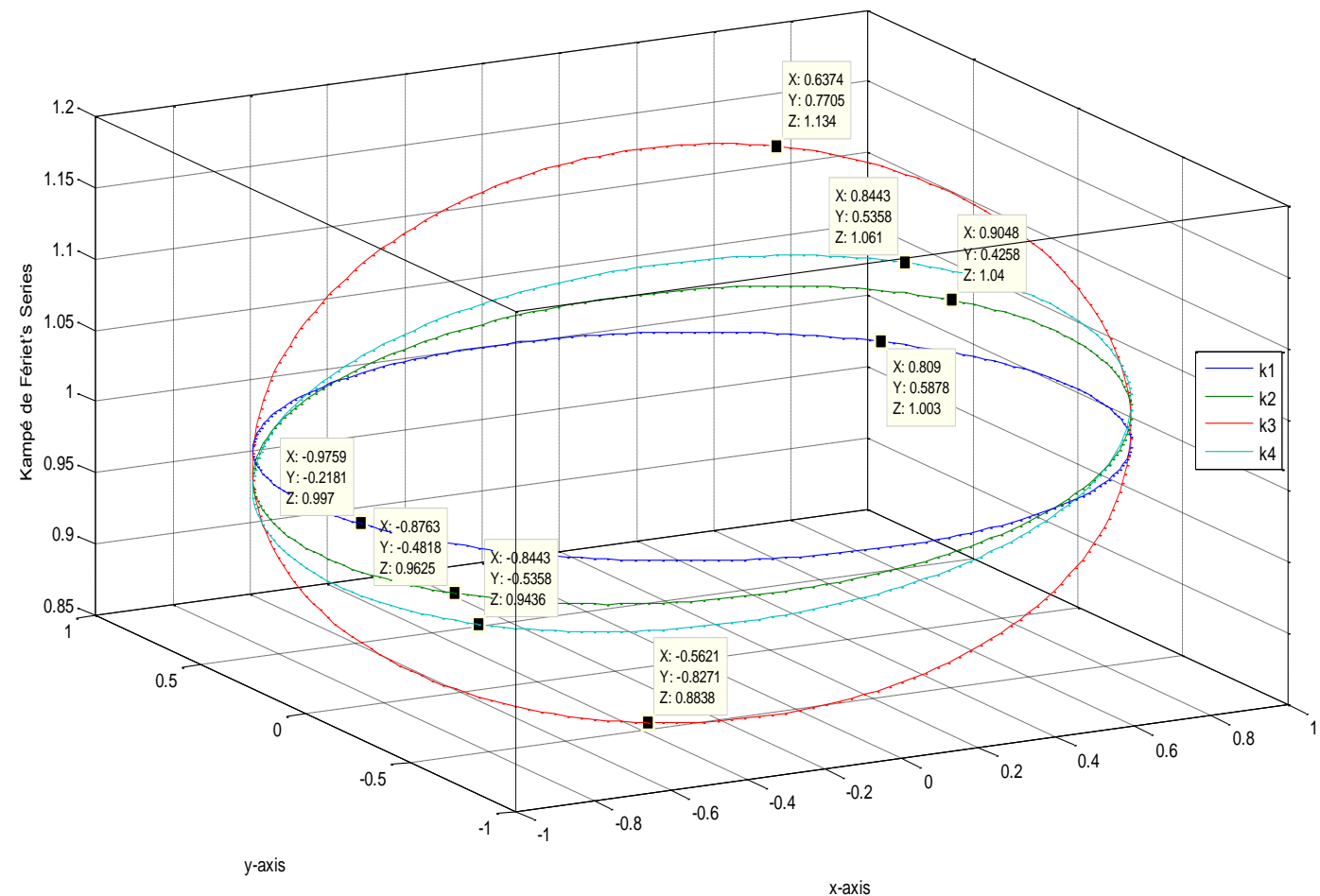

Fig. 8 


\section{INTERPRETATION OF DATA-BASE AND GRAPHS:}

The different values of the parameters, which we assigned for equation (2) and (3) are taken in command window in section (3) for different databases.

(I)

Database of $\quad F_{3: 33}^{33: 3}\left[\begin{array}{l}\left(a_{3}\right):\left(b_{3}\right) ;\left(c_{3}\right) ; \\ \left(\alpha_{3}\right):\left(\beta_{3}\right) ;\left(\gamma_{3}\right) ;\end{array}\right]$ for
$-5 \leq x, y \leq 5$ and different parameters are taken in Table (1). For $a_{1}=a_{2}=a_{3}=b_{1}=b_{2}=b_{3}$ $=c_{1}=c_{2}=c_{3}=\alpha_{1}=\alpha_{2}=\alpha_{3}=\beta_{1}=\beta_{2}=\beta_{3}=\gamma_{1}$ $=\gamma_{2}=\gamma_{3}$ the values of $\mathrm{K} 1, \mathrm{~K} 2, \mathrm{~K} 3$ and $\mathrm{K} 4$ remain the same and corresponding graph in fig. (1) of database (1) collides.

(II) Database of $\quad F_{33 ; 3 ;}^{33: 3}\left[\begin{array}{c}\left(a_{3}\right):\left(b_{3}\right) ;\left(c_{3}\right) ; \\ \left(\alpha_{3}\right):\left(\beta_{3}\right) ;\left(\gamma_{3}\right) ;\end{array}\right], y$ for $-5 \leq x, y \leq 5$ and different parameters are taken in Table (2). If $a_{1}=a_{2}=a_{3}, b_{1}=b_{2}=b_{3}, c_{1}=c_{2}=c_{3}$, $\alpha_{1}=\alpha_{2}=\alpha_{3}, \beta_{1}=\beta_{2}=\beta_{3}, \gamma_{1}=\gamma_{2}=\gamma_{3}$ the values of $\mathrm{K} 1, \mathrm{~K} 2$ and $\mathrm{K} 3$ in the interval $-5 \leq x, y \leq 0$ are $\mathrm{k} 3 \geq \mathrm{k} 2 \geq \mathrm{k} 1$ and on the other hand the values of $\mathrm{K} 1, \mathrm{~K} 2$ and $\mathrm{K} 3$ in the interval $0 \leq x, y \leq 5$ are $\mathrm{k} 1 \geq \mathrm{k} 2 \geq \mathrm{k} 3$ which can be seen and interpreted in the fig.(2).

(III) Database $\quad$ of $\quad F_{3: 3 ; 3}^{3: 33}\left[\begin{array}{l}\left.\left(a_{3}\right):\left(b_{3}\right) ;\left(c_{3}\right) ; x, y\right):\left(\beta_{3}\right) ;\left(\gamma_{3}\right) ; \\ \left(\alpha_{3}\right)\end{array}\right]$ for $-5 \leq x, y \leq 5$ and different parameters are taken in Table (3). For different parameters taken as in Table (3), the values of $\mathrm{K} 1, \mathrm{~K} 2, \mathrm{k} 3$ and $\mathrm{K} 4$ in the interval $-5 \leq x, y \leq 0$ are $\mathrm{k} 3 \geq \mathrm{k} 4 \geq \mathrm{k} 2 \geq \mathrm{k} 1$ and on the other hand the values of $\mathrm{K} 1, \mathrm{~K} 2, \mathrm{k} 3$ and $\mathrm{K} 4$ in the interval $0 \leq x, y \leq 5$ are $\mathrm{k} 1 \geq \mathrm{k} 2 \geq \mathrm{k} 4 \geq \mathrm{k} 3$ which can be seen and interpreted in fig. (3).

(IV) Database $\quad$ of $\quad F_{3: 2 ; 3}^{2: 3 ; 2}\left[\begin{array}{l}\left.\left(a_{2}\right):\left(b_{3}\right) ;\left(c_{2}\right) ; x, y\right):\left(\beta_{2}\right) ;\left(\gamma_{3}\right) ; \\ \left(\alpha_{3}\right)\end{array}\right]$ for $-1 \leq x, y \leq 1$ and different parameters are taken in Table (4). For $a_{1}=a_{2}=b_{1}=b_{2}=b_{3}=c_{1}=c_{2}=$ $\alpha_{1}=\alpha_{2}=\alpha_{3}=\beta_{1}=\beta_{2}=\gamma_{1}=\gamma_{2}=\gamma_{3}$ the values of $\mathrm{K} 1, \mathrm{~K} 2, \mathrm{k} 3$ and $\mathrm{K} 4$ in the interval $-5 \leq x, y \leq 0$ are $\mathrm{k} 1 \geq \mathrm{k} 2 \geq \mathrm{k} 3 \geq \mathrm{k} 4$ and on the other hand the values of $\mathrm{K} 1, \mathrm{~K} 2, \mathrm{k} 3$ and $\mathrm{K} 4$ in the interval $0 \leq x, y \leq 5$ are $\mathrm{k} 4 \geq \mathrm{k} 3 \geq \mathrm{k} 2 \geq \mathrm{k} 1$. Graphs of $\mathrm{k} 3$ and $\mathrm{k} 4$ for $-5 \leq x, y \leq 0$ approximately collides, graph of $\mathrm{k} 2$, $\mathrm{k} 3$ and $\mathrm{k} 4$ for $0 \leq x, y \leq 5$ approximately collides, which can be seen and interpreted in the fig.(4).

(V) Database of $\quad F_{3: 2 ; 3}^{2: 3 ; 2}\left[\begin{array}{l}\left.\left(a_{2}\right):\left(b_{3}\right) ;\left(c_{2}\right) ; x, y\right):\left(\beta_{2}\right) ;\left(\gamma_{3}\right) ; \\ \left(\alpha_{3}\right)\end{array}\right]$ for $-10 \leq x, y \leq 10$ and different parameters are taken in Table (5). The values of $\mathrm{K} 1, \mathrm{~K} 2, \mathrm{k} 3$ and $\mathrm{K} 4$ in the interval $-10 \leq x, y \leq 0$ are $\mathrm{k} 3 \geq \mathrm{k} 4 \geq \mathrm{k} 2 \geq \mathrm{k} 1$ and on the other hand the values of $\mathrm{K} 1, \mathrm{~K} 2, \mathrm{k} 3$ and $\mathrm{K} 4$ in the interval $0 \leq x, y \leq 10$ are $\mathrm{k} 1 \geq \mathrm{k} 2 \geq \mathrm{k} 4 \geq \mathrm{k} 3$, which can be seen and interpreted in the fig. (5). If the graphs in figure (2), (3), (4) and (5) compared, approximately the graphs are same for different parameters.

(VI) In all the cases of data-base for each interval of $\mathrm{x}$ and $\mathrm{y}$, the values of $\mathrm{K} 1, \mathrm{~K} 2, \mathrm{~K} 3, \mathrm{~K} 4$ only at the points $(-5,-5),(-4,-4), \ldots,(5,5)$ are obtained using Matlab. In section (5), Mesh-Plot graphs for the interval and values of parameters taken as in section (5) in command window (a) and (b). In fig.(6), when $\mathrm{x}=4, \mathrm{y}=4, \mathrm{~K} 1=1.012 ; \mathrm{x}=3, \mathrm{y}=-2, \mathrm{~K} 1=0.9991$; $\mathrm{x}=-2, \mathrm{y}=3, \mathrm{~K} 1=1.004 ; \mathrm{x}=-4, \mathrm{y}=-1, \mathrm{~K} 1=0.9942 ; \mathrm{x}=-$ $3, y=-4, K 1=0.9895$. In fig.(7), when $x=4, y=4$, $\mathrm{K} 1=1.016 ; \mathrm{x}=2, \mathrm{y}=-2, \mathrm{~K} 1=1.004 ; \mathrm{x}=-2, \mathrm{y}=-2, \mathrm{~K} 1=$ $0.9923 ; \quad \mathrm{x}=-4, \quad \mathrm{y}=2, \quad \mathrm{~K} 1=0.991 ; \quad \mathrm{x}=-5, \quad \mathrm{y}=5$, $\mathrm{K} 1=0.9914$. In mesh plots, when $\mathrm{x} \neq \mathrm{y}$ at each order pair of values of Kampé de Fériet's Series can be easily interpreted.

(VII) In section (6), we plot the graph for circular points of unit circle taken as $\mathrm{x}=\cos (\mathrm{t})$, $\mathrm{y}=\sin (\mathrm{t})$ where $t=-\pi: \pi / 100: \pi$ for $\mathrm{k} 1, \mathrm{~K} 2, \mathrm{~K} 3$ and $\mathrm{K} 4$ taken as in section (6) for different parameters. We can see that $\mathrm{K} 1$ has maximum value 1.003 and minimum value 0.997 ; K2 has maximum value 1.04 and minimum value 0.9436 ; $\mathrm{K} 3$ has maximum value 1.134 and minimum value 0.8838 $\mathrm{K} 4$ has maximum value 1.061 and minimum value 0.9436 .

\section{CONCLUSION:}

Computing the Kampé de Fériet's Series is an important problem due to its wide variety of applications in problems in mathematical and theoretical physics and many other areas. This paper presents several cases of Matlab applications in mathematical analysis including generating the graph and database of Kampé de Fériet's Series. Program made for Kampé de Fériet's Series is easy to establish and implementation for Graphs and Data-Bases. From the table (1) and Fig. 1, it is easily interpreted that for equal values of parameters, values of Kampé de Fériet's Series are identical. Fig. 2, 3, 4 and 5 interprets that the graph of Kampé de Fériet's Series are approximately same for different values of the parameters. In section (5), Mesh-Plot is an interesting graph from which at every pair of $\mathrm{x}$ and $\mathrm{y}$ in the given range, the values of Kampé de Fériet's Series can be easily taken. Graph for circular points of Kampé de Fériet's Series interpret for small interval $(-1,1)$ both for $\mathrm{x}$ and $\mathrm{y}$ variable for different parameters. Reader can take any value of the parameters for any range of $\mathrm{x}$ and $\mathrm{y}$ to analyze the Kampé de Fériet's Series.

\section{REFERENCES}

[1] Askey, R. A.; Daalhuis, Adri B. Olde (2010), "Generalized hypergeometric function", in Olver, Frank W. J.; Lozier,Daniel M.; Boisvert, Ronald F. et al., NIST Handbook of Mathematical Functions, Cambridge University Press, ISBN 978-0521192255. 
[2] Bevel, Tom; Gardner, Ross M. (2008) Bloodstain Pattern Analysis With an Introduction to Crimescene Reconstruction, 3rd Ed. CRC Press.

[3] Daalhuis, Adri B. Olde (2010), "Hypergeometric function", in Olver, Frank W. J.; Lozier, Daniel M.; Boisvert, Ronald F. et al., NIST Handbook of Mathematical Functions, Cambridge University Press. Desmond J. Higham, Nicholas J. Higham (2005), MATLAB guide / SIAM.

[4] Dwork, B(1990), Generalized Hypergeometric Functions. Oxford, England: Clarendon Press.

[5] Exton, H. and Krupnikov, E. D. A Register of ComputerOriented Reduction Identities for the Kampé de Fériet Function. Draft manuscript. Novosibirsk, 1998

[6] H.M. Srivastava and P.W. Karlsson, Multiple Gaussian Hypergeometric Series (Ellis Horwood Ltd., Chichester, U.K., 1985).

[7] H.M. Srivastava and H.L. Manocha, A Treatise on Generating Functions (Ellis Horwood Ltd., Chichester, U.K., 1984).

[8] H.M. Srivastava and R. Panda, Some bilateral generating functions for a class of generalized hypergeometric polynomials. J. Reine Angew. Math., 283/284, 265-74.

[9] H. Exton, Handbook of Hypergeometric Integrals (Ellis Horwood Ltd., Chichester, U.K., 1978).
[10] Hui Wang, Saumuy Suriano and Liang Zhou (2009), "High definition metrology based spatial variation pattern analysis for machining process monitoring and diagnosis", ASME 2009 International Manufacturing Science and Engineering Conference/ West Lafayette, Indiana (USA)/ Volume 2, ISBN: 978-0-7918-4362-8/ Paper no. MSEC2009-84154 pp.471-480.

[11] Rumshisky, Anna, and Pustejovsky, James (2006), "Inducing Sense-Discriminating Context Patterns from Sense-Tagged Corpora". LREC, Genoa, Italy.

[12] Stormy Attaway (2009), “MATLAB: a practical introduction to programming and problem solving"/Butterworth-Heinemann.

[13] Watanabe Y. and Takahashi K. (1998), "A fast structural matching and its application to pattern analysis of 2-D electrophoresis images"/ Image Processing, 1998. ICIP 98. Proceedings. International Conference / page(s): 804 -808 vol.3.

[14] Yoshida, Masaaki (1997). Hypergeometric Functions, My Love: Modular Interpretations of Configuration Spaces. Braunschweig/Wiesbaden: Friedr. Vieweg \& Sohn. MR1453580.ISBN 3-528-06925-2. 